\title{
Hierarchical architecture as a new approach for building web applications
}

\begin{abstract}
Controlling and testing a large scale web application is a time consuming and expensive job because of the huge number of pages and their actions. The hierarchical architecture proposed in this paper is a framework which can decreases nested relations between web pages. We can also use different programming methods like object oriented or structured over this model. One of the most important advantages of this architecture is increasing the control and security of the application in its different layers. This architecture also eases adding new modules to the main body of the program and software maintenance.
\end{abstract}

Keyword: Software engineering; Web application; Portal; Hierarchical architecture; Security 